\title{
Control of Widely Tunable SSG-DBR Lasers for Dense Wavelength Division Multiplexing
}

\author{
Gert Sarlet, Student Member, IEEE, Geert Morthier, Member, IEEE, and \\ Roel Baets, Senior Member, IEEE, Member, OSA
}

\begin{abstract}
This paper presents a general discussion on the control of widely tunable super structure grating distributed Bragg reflector (SSG-DBR) lasers. A feedback control scheme is presented that ensures frequency stability and accuracy (better than \pm 0.5 $\mathrm{GHz})$, as well as high side mode suppression ratio $(>35 \mathrm{~dB})$. The active section voltage is monitored to maintain mode stability and a highly stable Fabry-Perot etalon is used as a reference to lock the laser frequency to a specific ITU channel. It is shown that stability can even be maintained when directly modulating the laser at $1.244 \mathrm{~Gb} / \mathrm{s}$. Furthermore, a characterization scheme is demonstrated that uses the voltage monitoring to generate a look-up table of operation points very efficiently and accurately. For all operation points, the frequency accuracy is better than $\pm 0.5 \mathrm{GHz}$ and the side mode suppression ratio is above $35 \mathrm{~dB}$.
\end{abstract}

Index Terms-Distributed Bragg reflector (DBR) lasers, laser stability, laser tuning, semiconductor lasers, wavelength division multiplexing (WDM).

\section{INTRODUCTION}

$\mathbf{T}$ UNABLE lasers are currently the focus of much attention in the telecom industry, as they are expected to become essential components for the next generation of dense wavelength division multiplexing (DWDM) systems. At first, tunable transmitters will probably be used as a backup for standard fixedwavelength transmitters, since network operators would then not have to maintain a large and costly inventory of spare transmitter cards, each dedicated to a single channel. On a longer term, tunable transmitters could however entirely replace fixedwavelength lasers, not only providing significant inventory savings but also simplifying equipment operation and maintenance.

Once tunable transmitters are installed, their inherent flexibility can of course be used to achieve optical networking functionality. For instance, in the WDM ring networks with fixedwavelength optical add/drop multiplexers (OADMs) that are already being deployed, the use of a tunable laser would allow network reconfiguration, dynamic bandwidth allocation and wavelength protection. However, also to realize more advanced alloptical networks, which use optical cross connects (OXCs), a tunable transmitter might be indispensable. With high channel numbers (more than 100) and low channel spacing (50 or 25 $\mathrm{GHz}$ ) the cost and complexity of an OXC operating on a single channel level become prohibitive. A feasible alternative would

Manuscript received January 14, 2000; revised May 2, 2000. This work was supported by the EU Project ACTS-AC329 ACTUAL. The work of G. Sarlet was supported by the Fund for Scientific Research-Flanders (Belgium).

The authors are with the Department of Information Technology, University of Gent-IMEC, Gent B-9000, Belgium.

Publisher Item Identifier S 0733-8724(00)06481-1. be to have the OXC route bands of channels and use tunable transmitters and receivers to address a particular channel within a given band.

Essential for all the above applications is that the laser can be tuned to a particular channel very accurately and reliably, which is largely dependent on the electronics and algorithms that are used to control the tunable laser. Typical requirements are a frequency accuracy better than $\pm 10 \%$ of the channel spacing, and a guaranteed side-mode suppression ratio (SMSR) of at least $30 \mathrm{~dB}$.

The classic tunable laser is the three-section distributed Bragg reflector (DBR) laser [1] [2]. The tuning range of these lasers is limited by the maximum refractive index change that can be achieved through current injection $(\Delta \lambda / \lambda=\Delta n / n)$. Typical tuning ranges are on the order of 5-10 nm, although devices with a tuning range of more than $15 \mathrm{~nm}$ have been demonstrated [3]. Several approaches have been proposed to overcome this limitation. In the grating coupler sampled reflector (GCSR) laser [4]-[6] a broadly tunable put poorly selective grating assisted codirectional coupler filter is combined with a periodically sampled Bragg grating, which exhibits a comb-shaped reflection spectrum [7]. Another method consists of using two periodically modulated Bragg reflectors, which have comb-shaped reflection spectra with slightly different peak spacing (Fig. 1), such that the tuning behavior is similar to the operation of a Vernier scale. The modulation can take two forms: a periodic sampling of the grating (sampled grating, SG) [8]-[9] or a periodic phase modulation of the grating (super structure grating, SSG) [10]-[11]. All these devices have demonstrated quasicontinuous tuning ranges of $40-60 \mathrm{~nm}$.

When it comes to controlling such devices, a distinction has to be made between two aspects. First of all one needs a look-up table, which tells what currents should be applied to the different sections to get a certain output power and frequency (with high SMSR). The current sources driving the laser will typically be controlled by a microprocessor, which reads the appropriate data from the look-up table (stored in an EEPROM) when output power and/or frequency should be changed. On the other hand, one typically also wants some sort of feedback control, which ensures long-term stability and frequency accuracy.

Because of fabrication tolerances, a look-up table has to be generated for every individual laser. Moreover, after some years of use, transmitters might need to be recalibrated because of aging, or the look-up table might have to be adjusted to changes in system specifications. It is therefore crucial that the characterization time (or look-up table generation time) is reduced as much as possible, in order to minimize cost. 


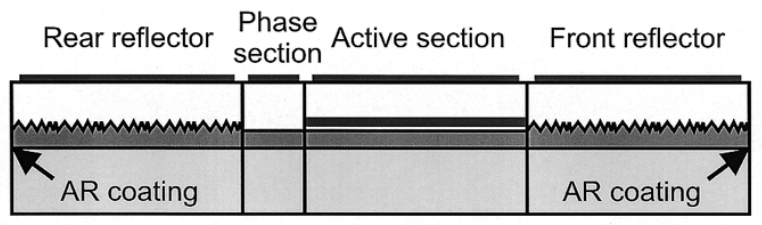

Fig. 1. Schematic cross-section of an SSG-DBR laser.

Feedback control loops can correct a possible frequency drift or reduction of mode stability due to device aging or varying environmental conditions. If the feedback control is handled by a microprocessor, the variation over time of the different control currents can be monitored. These data could be used to decide when a recalibration of the device is in order. Alternatively, one could try to develop a scheme that would update the look-up table online, based on the evolution of the control currents for a single channel.

In this paper, we focus on one particular type of widely tunable laser, the SSG-DBR laser, although with minor adjustments the methods described should also apply to other DBR-type tunable lasers. The SSG-DBR lasers used in the different experiments were all fabricated by NTT. A feedback control scheme is presented, which ensures frequency stability and accuracy, as well as high side mode suppression ratio. The active section voltage is monitored to maintain mode stability and a highly stable Fabry-Perot etalon is used as a reference to lock the laser frequency to a specific ITU channel. It is shown that stability can even be maintained when directly modulating the laser at $1.244 \mathrm{~Gb} / \mathrm{s}$. Furthermore, a characterization method is demonstrated that uses the voltage monitoring to generate a look-up table of operation points very efficiently and accurately.

\section{OPERATION PRINCIPLE OF THE SSG-DBR LASER}

A schematic of an SSG-DBR laser is shown in Fig. 1. The two SSG reflectors have comb-shaped reflection spectra, with slightly different peak spacing. For stable single-mode operation, a peak of each reflection comb and a longitudinal mode have to be aligned (Fig. 2). The SSG reflectors can be tuned by current injection. A typical tuning characteristic is shown in Fig. 3. If one of the reflectors is tuned, different pairs of reflector peaks will successively overlap and the lasing frequency will jump by approximately the peak spacing (coarse tuning). Medium tuning is obtained by shifting both reflectors simultaneously. The frequency will then show smaller jumps, typically about $50 \mathrm{GHz}$, corresponding to the cavity mode spacing. For fine tuning, the longitudinal modes are shifted by injecting current into the phase section. Consequently, by selecting an appropriate combination of front DBR, rear DBR and phase current, the laser can be tuned to any frequency within a range of a few $\mathrm{THz}$ (quasicontinuous tuning).

\section{FeEdBack CONTROL-CONCEPT}

\section{A. Frequency Stabilization}

As mentioned above, fine tuning of the SSG-DBR laser is done by changing the current through the phase section. A feedback signal for frequency stabilization can be generated

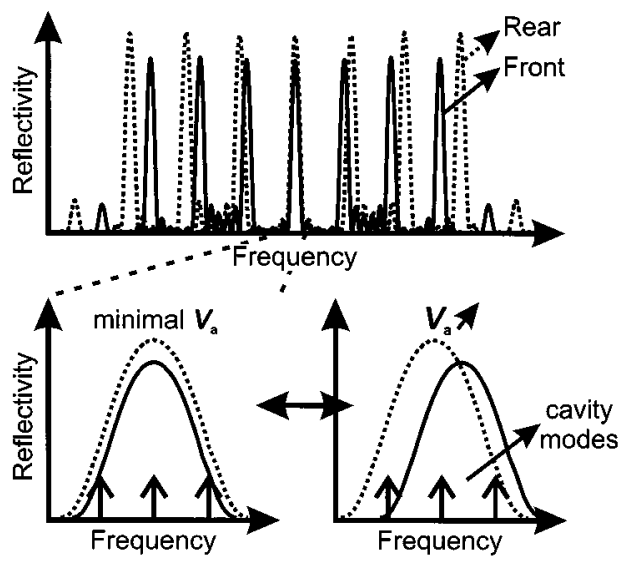

Fig. 2. Reflectivity spectra of front and rear SSG reflectors. Stable operation points with good SMSR are obtained when a cavity mode is aligned with a peak of each reflector (bottom right).

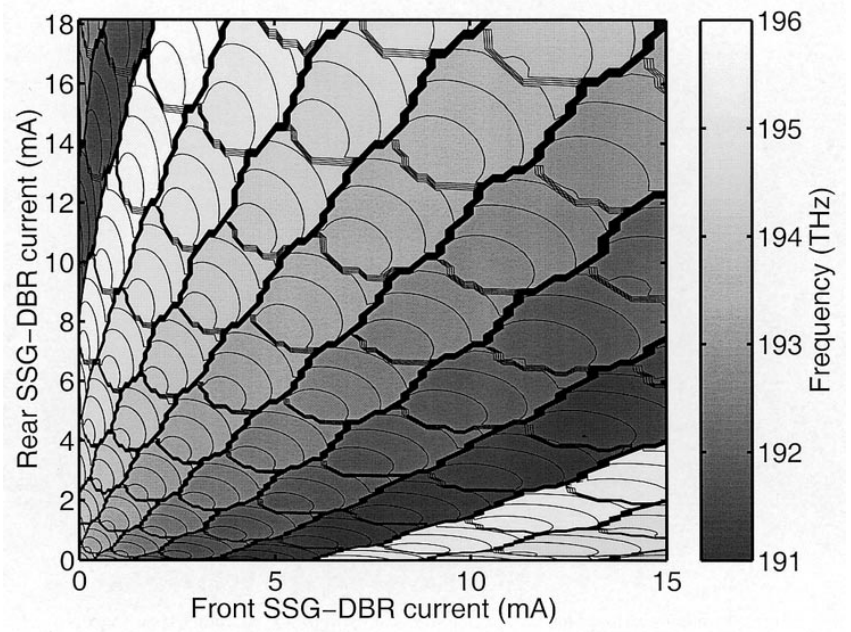

Fig. 3. Calculated tuning characteristic of a typical SSG-DBR laser, showing laser frequency as a function of the two reflector currents. Frequency contours are plotted in $10-\mathrm{GHz}$ increments.

using a reference optical filter. Examples of such filters are: a Fabry-Perot etalon [12], a fiber Bragg grating [13]-[14] or an arrayed waveguide grating (AWG) [15]. For test purposes, the operation of such a filter can be simulated using a PC and the signal from a wavelength meter or an optical spectrum analyzer [16]. In all cases the resulting signal is used to adjust the phase section current, such that the frequency remains locked to the external reference.

\section{B. Mode Stabilization}

When only a frequency control loop as just described is applied, there is no guarantee that a high SMSR will be maintained. If the laser were to drift for some reason and only the phase current would be adjusted to correct the frequency, eventually a mode hop would occur [15]-[16]. Indeed, at some point the initial cavity mode would have moved so far from the reflectivity peaks that an adjacent cavity mode would become the preferential mode. Therefore, a second control loop is needed that keeps the two reflector peaks aligned with the selected cavity mode. 


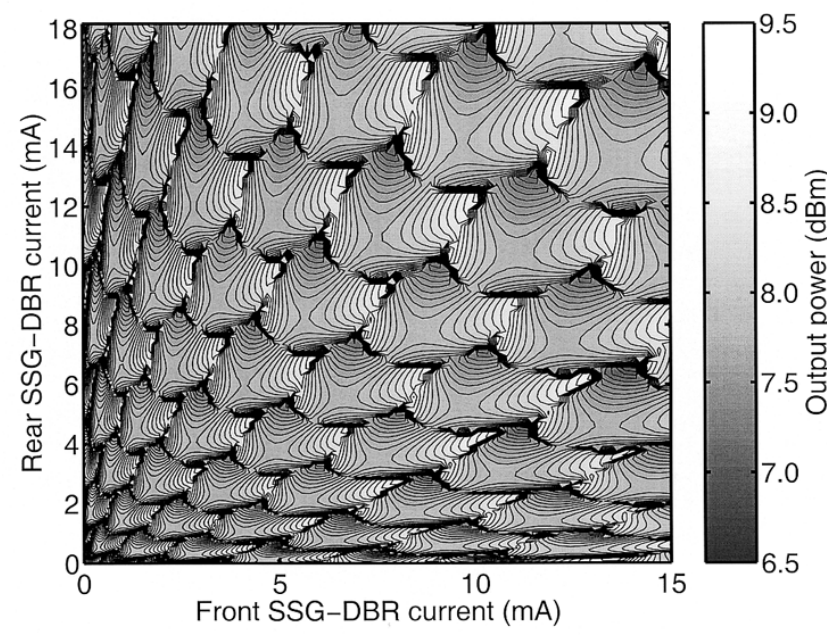

(a)

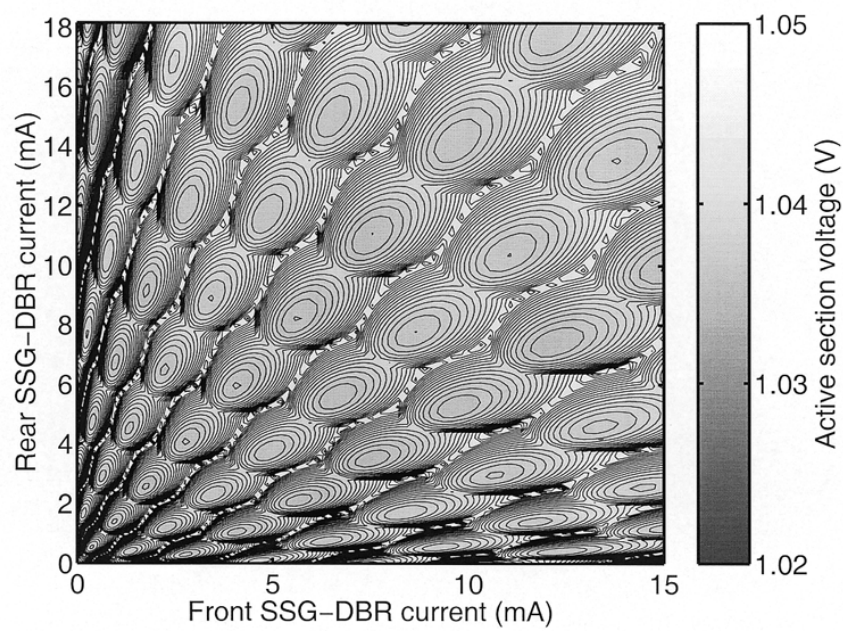

(b)

Fig. 4. Calculated contour maps of output power (a) and active section voltage (b) as a function of front and rear reflector current. Carrier-induced losses were neglected in the passive sections.

For simple three-section DBR lasers, a correlation was observed between the SMSR and the variation of output power with tuning current. This has been used to develop a control loop that ensures high SMSR [17]. Analogous observations were made for SSG-DBR lasers by Ishii et al. [15]: a high SMSR can be maintained by locking to a saddle point in the output power (with respect to the front and rear reflector currents). However, as was shown in [16], carrier-induced losses in the reflector sections limit the applicability of this approach.

Fig. 4(a) shows the variation of the output power as a function of the reflector currents when carrier-induced losses in the reflector sections are neglected. In the center of each mode region (cf., Fig. 3) a saddle point is observed: a minimum with respect to the front DBR current coincides with a maximum with respect to the rear DBR current. At these locations, optimal alignment of a cavity mode with a reflection peak of each DBR is obtained and therefore a high SMSR is assured. Still, in practice there will always be some carrier-induced losses. In Fig. 5(a) a contour map of output power as a function of the reflector currents is plotted, which was calculated using a typical

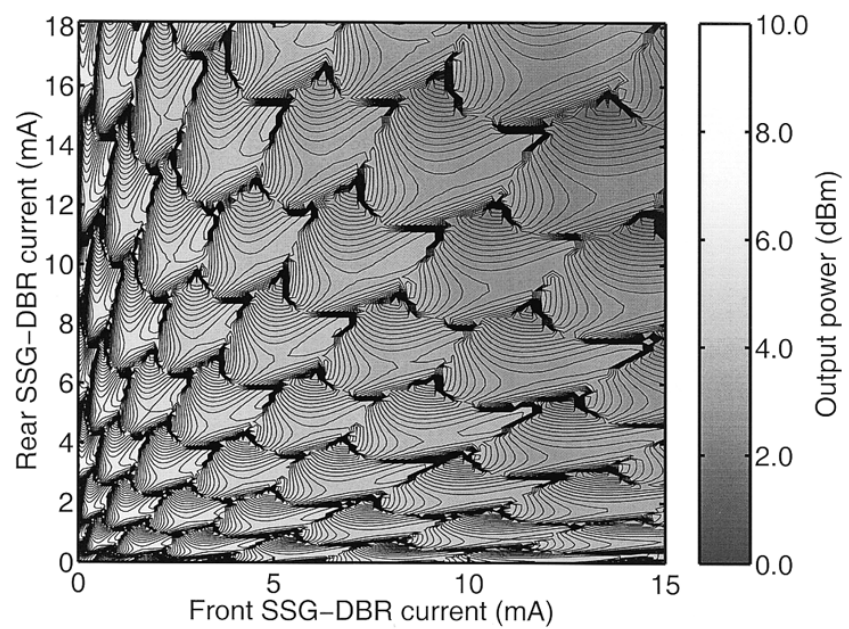

(a)

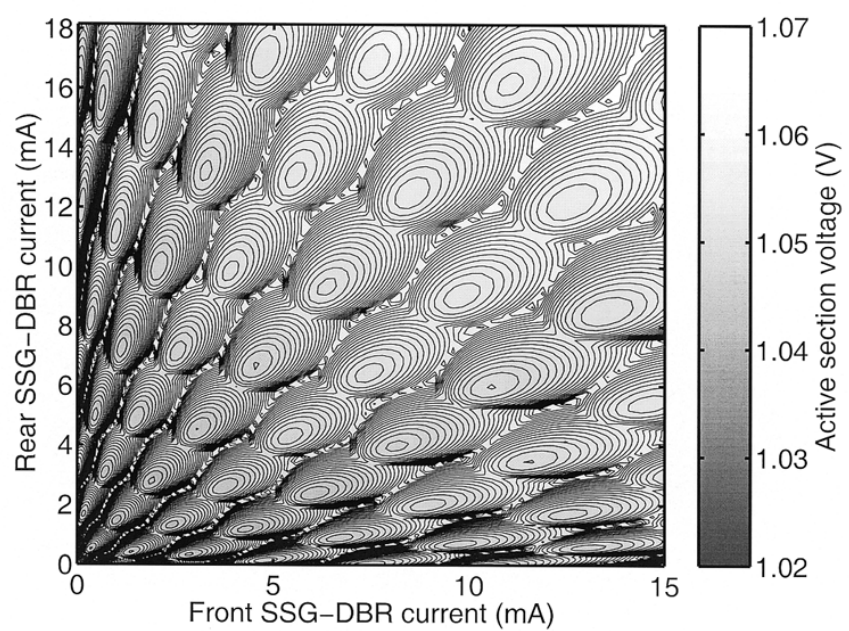

(b)

Fig. 5. Calculated contour maps of output power (a) and active section voltage (b) as a function of front and rear reflector current. For the passive sections, a typical value was assumed for the increase of the loss per unit length $\alpha$ with carrier density $N\left(d \alpha / d N=3.0 \times 10^{-17} \mathrm{~cm}^{2}\right)$.

value for the increase of the loss per unit length $\alpha$ with carrier density $N\left(d \alpha / d N=3.0 \times 10^{-17} \mathrm{~cm}^{2}\right)$. Clearly, the saddle points have shifted toward lower rear and higher front DBR currents, and have even disappeared at high currents. The effect is of course largest for the front reflector, since the output light has to pass through this section. This severely limits the application range of the feedback loop described in [15]. One might consider correcting the measured power values for the carrier-induced losses, but this does not seem very practical, especially since there is no guarantee that the needed correction will not vary over the lifetime of the device.

Fortunately, there is an alternative signal that is equally easy to measure, that is also correlated to the SMSR, but is less sensitive to the carrier-induced losses: the voltage across the active section. In the loss-less case, the perfect alignment of the reflector peaks with a cavity mode corresponds to a (local) minimum in threshold gain and carrier density [15], since this cavity mode will then see a maximal reflectivity from both ends. Moving one of the reflectors out of alignment will increase the 


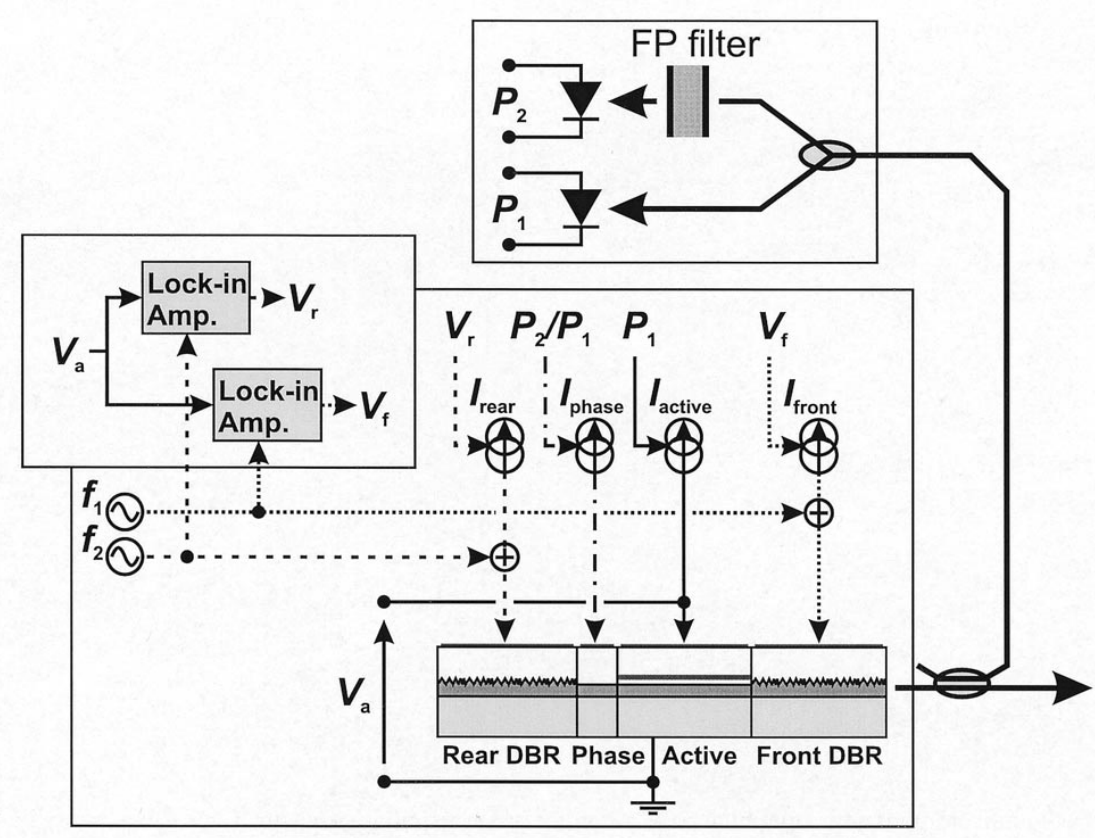

Fig. 6. Schematic of control circuit for frequency, mode and power stabilization of an SSG-DBR laser.

threshold gain and the carrier density (Fig. 2). Since the active section voltage (at fixed bias current) depends on the carrier density, it will also increase. Fig. 4(b) shows the variation of active section voltage with tuning in the loss-less case. Clearly, minima in active section voltage appear at the same locations where saddle points in output power are observed. If the carrier-induced losses are taken into account, the minima in voltage shift toward lower front and rear DBR currents. However, as Fig. 5(b) proves, all minima remain well within the mode boundaries and minima are still observed for modes where the saddle point in the output power has disappeared. This clearly explains the experimental results previously presented in [16].

\section{FEEDBACK CONTROL-EXPERIMENTS}

\section{A. Static Operation}

Fig. 6 shows a schematic of the control circuit for frequency, mode and power stabilization that was built. In the set-up, all currents are controlled by a program running on a PC. Currents are adjusted by communicating with the current sources via GPIB. This makes the feedback loop relatively slow, but has the advantage that the currents can be monitored at all times. Additionally, the program can interrupt the operation of the loop at regular intervals to measure frequency, output power and SMSR and store these measurement results together with the values of the different currents. Initial operation points for all channels on the 100-GHz ITU grid are stored in a look-up table.

Three different feedback loops can be distinguished. For mode stabilization, small modulation signals (typically $5 \mu \mathrm{A}$ ) are added to the front and rear reflector currents, at frequencies $f_{1}$ and $f_{2}$ respectively. With two lock-in amplifiers, the in-phase modulation components $V_{f}$ and $V_{r}$ of the active section voltage $V_{a}$ at these frequencies are measured. At each iteration of the feedback loop, a correction proportional to $V_{f}\left(V_{r}\right)$ is added to the front (rear) reflector current. In this way, the laser is stabilized at a local minimum in active section voltage (with respect to the reflector currents).

For frequency control, a commercially available wavelength locker is used [18]. In this component, a fraction $(\approx 5 \%)$ of the light emitted from the front facet is tapped off. This light is then split into two parts, of which one is directly detected (power $P_{1}$ ) and the other is first filtered through a highly stable Fabry-Perot etalon with a free spectral range (FSR) of $100 \mathrm{GHz}$ (power $P_{2}$ ). If $\log \left(\eta P_{2} / P_{1}\right)$ is plotted as a function of frequency around an ITU grid frequency, a fairly linear response curve which crosses zero at the grid frequency is obtained $(\eta \approx 1.32$ is a calibration factor). Consequently, corrections proportional to $\log \left(\eta P_{2} / P_{1}\right)$ are added to the phase section current.

Finally, for power stabilization, the unfiltered power $P_{1}$ is fed back to the active section current.

The feedback control setup was tested for all channels on the 100-GHz ITU grid the laser could be tuned to, a total of 41 channels with frequencies ranging from 191.9 to $195.9 \mathrm{THz}$. In order to cause some frequency drift, the temperature of the laser submount was changed from 20 to $30^{\circ} \mathrm{C}$. After stabilization at each temperature, the frequency, SMSR and output power were measured. As Fig. 7 illustrates, the frequency standard deviation was below $0.2 \mathrm{GHz}$ for all channels except $192.4 \mathrm{THz}$ (the maximum frequency variation was less than $1.0 \mathrm{GHz}$ ). For all channels an SMSR of more than $35 \mathrm{~dB}$ was maintained, except again for the 192.4-THz channel where the SMSR decreased for temperatures above $28{ }^{\circ} \mathrm{C}$ (Fig. 8). The output power was stabilized at $0.5 \mathrm{~mW}$ and as Fig. 7 shows, very small power variations were measured.

The larger frequency errors and the decreasing SMSR for the $192.4 \mathrm{THz}$ channel are explained by a thermal runaway effect. At $20^{\circ} \mathrm{C}$ this channel requires both a high front and a high rear DBR current, 24.6 and 16.6 mA, respectively (Fig. 9). Because 


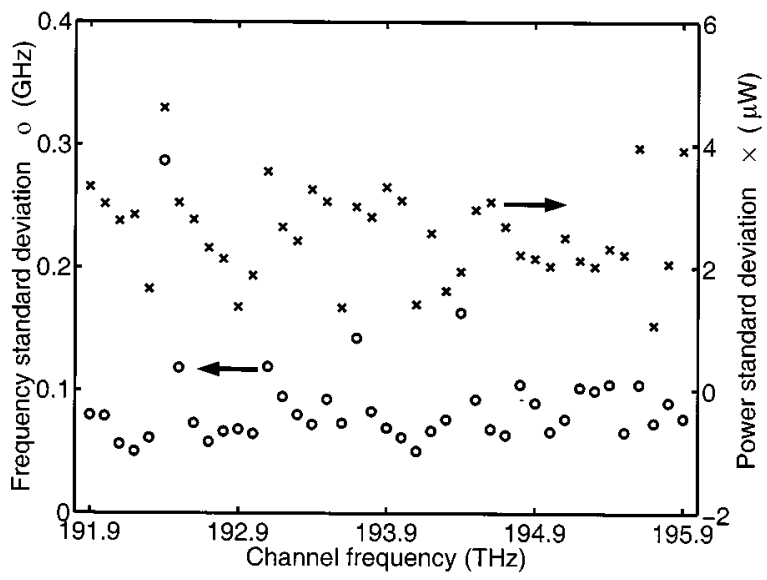

Fig. 7. Frequency standard deviation and power standard deviation for 41 ITU channels for a temperature sweep from 20 to $30^{\circ} \mathrm{C}$. The output power was stabilized at $0.5 \mathrm{~mW}$.

of the carrier-induced losses in the tuning sections, this means that also a high active section current is needed to maintain an output power of $0.5 \mathrm{~mW}$. Due to the nonzero thermal resistance between the laser waveguide and the submount, the waveguide temperature is always higher than the submount temperature and the difference is more or less proportional to the injected current. When the submount temperature is ramped up, the phase current has to be increased to counteract the thermal tuning. At the same time, the reflector currents are raised such that the reflector peaks track the cavity mode. This causes higher loss in all three passive sections and as a consequence, also the active section current has to be boosted. From Fig. 9, it is clear that the currents increase superlinearly, since the tuning efficiency decreases with current due to Auger recombination and heating effects. All this amounts to a lot of extra heat being generated in the laser waveguide. At a certain point, the waveguide temperature rise due to a further increase in current completely counteracts the electronic tuning and therefore frequency and mode stability are degraded. Note, however, that at temperatures above $25^{\circ} \mathrm{C}$ there is an alternative combination of front DBR, rear DBR and phase currents that will give the same output frequency with much lower currents.

In Fig. 7, frequency standard deviations were plotted in an attempt to eliminate the errors due to the intrinsic accuracy of the wavelength locker. The commercial wavelength locker [18] used in these experiments has a specified channel accuracy of $\pm 2.5 \mathrm{GHz}$, and a temperature stability of $\pm 1.0 \mathrm{GHz}\left(0^{\circ} \mathrm{C}\right.$ to $70{ }^{\circ} \mathrm{C}$, no internal temperature control). By properly choosing the calibration factor $\eta$ in the $\log \left(\eta P_{2} / P_{1}\right)$ relation, we have however achieved much better accuracies. The circles in Fig. 10 show the true frequency errors after stabilization at a submount temperature of $20^{\circ} \mathrm{C}$, with a $\eta$-value of 1.32 . Clearly, all channels are within $\pm 0.5 \mathrm{GHz}$ of the specified frequency. Note also that the wavelength meter used here (HP 86120B multiwavelength meter) has a resolution of only $0.1 \mathrm{GHz}$, and a specified absolute frequency accuracy of $\pm 3 \mathrm{ppm}( \pm 0.6 \mathrm{GHz}$ ) [19].

A possible disadvantage of the control circuit is the fact that small sinusoidal signals $(5 \mu \mathrm{A})$ are added to the front and rear reflector currents. Since the average tuning efficiency of the re-

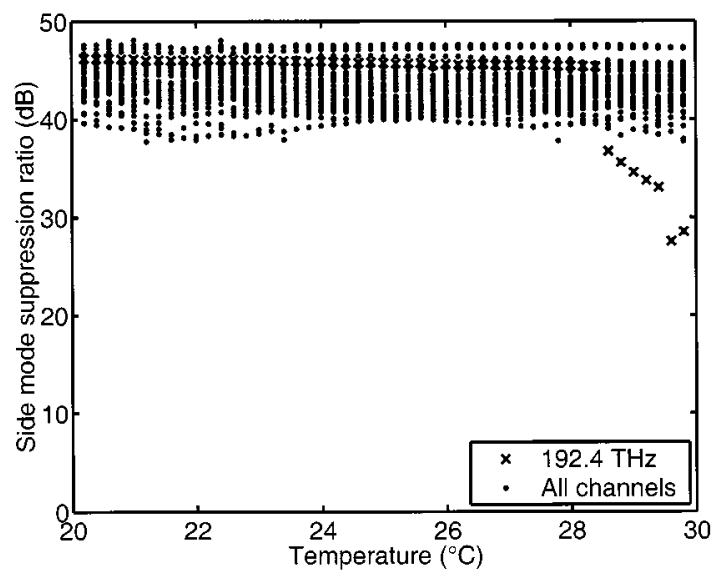

Fig. 8. Variation of side mode suppression ratio for 41 ITU channels for a temperature sweep from 20 to $30^{\circ} \mathrm{C}$. Crosses $(x)$ indicate the worst case channel at $192.4 \mathrm{THz}$

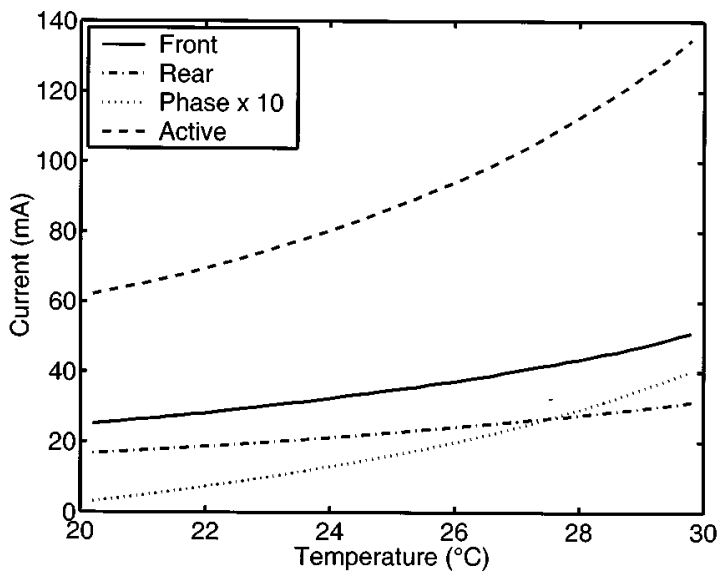

Fig. 9. Variation of drive currents with temperature for the channel at 192.4 THz.

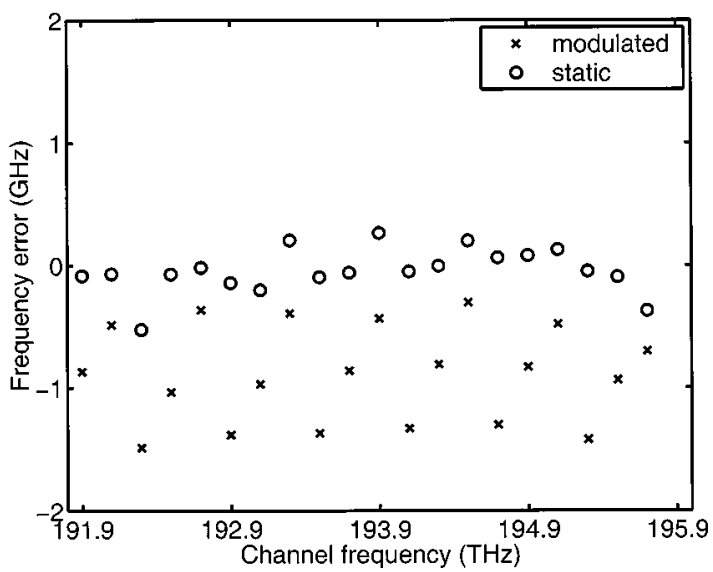

Fig. 10. Frequency errors after stabilization of 20 ITU channels at $20^{\circ} \mathrm{C}$, with direct modulation at $1.244 \mathrm{~Gb} / \mathrm{s}(\times)$ and in static operation (o).

flection peaks of the DBR sections is $25 \mathrm{GHz} / \mathrm{mA}$, the modulation of the reflection peaks is approximately $125 \mathrm{MHz}$. The corresponding variation of the laser frequency, or in other words the variation of the cavity mode frequency, is however only a fraction of this value, typically 0.2 [15]. With current modulation 
on both reflectors, this gives a total frequency modulation on the order of $50 \mathrm{MHz}$. At the same time, a slight power modulation is added. Measurements have shown however that relative power variations are very small, on the order of $10^{-10}$ to $10^{-9}$. It has to be noted that these frequency and power fluctuations are much slower than typical data rates, so the variation during a single bit period will be negligible.

\section{B. Operation with Direct Modulation}

The frequency and mode control circuits were also tested while the laser was directly modulated. A $2^{23}-1$ PRBS signal with a current swing of $60 \mathrm{~mA}$ was applied to the active section. The bit rate was chosen at $1.244 \mathrm{~Gb} / \mathrm{s}$ because parasitics in the electrical path limited the small signal bandwidth to approximately $800 \mathrm{MHz}$. Recent experiments have shown however that transmission over $80 \mathrm{~km}$ of standard single-mode fiber is possible with an SSG-DBR laser (with proper electrical contacting) directly modulated at $2.488 \mathrm{~Gb} / \mathrm{s}$ [20]. The $2^{23}-1$ PRBS signal has a fundamental frequency component

$$
f_{0}=\frac{1.244 \times 10^{9}}{2^{23}-1} \mathrm{~Hz}=148.3 \mathrm{~Hz}
$$

For the mode stabilization circuit to operate properly the modulation frequencies of the reflector currents ( $f_{1}$ and $f_{2}$, cf., Fig. 6) have to be chosen away from $f_{0}$ and its higher harmonics. The noise on the signals $V_{f}$ and $V_{r}$ is also lower at low modulation frequencies. Therefore, we took $f_{1}=1410 \mathrm{~Hz}$ and $f_{2}=960$ $\mathrm{Hz}$. To increase the signal-to-noise ratio (SNR), the modulation amplitude was increased to $25 \mu \mathrm{A}$. Furthermore, a larger time constant was chosen for the lock-in amplifier. With a true random bit signal, some bandpass-filtering might be required in front of the lock-in amplifiers.

Tests were performed for 20 channels at $200 \mathrm{GHz}$ spacing, from 191.9 to $195.7 \mathrm{THz}$. The laser was roughly tuned to the appropriate channel. Subsequently, the active section bias current was adjusted manually in order to get both a good extinction ratio (typically $11 \mathrm{~dB}$ ) and a good SMSR. The value of the active section bias current is quite critical. It should be close to 30 $\mathrm{mA}$ above the threshold value for the selected channel. A too low value will reduce the SMSR and cause increased turn-on jitter, whereas a too high value will reduce the extinction ratio (ER).

After setting the bias current, the frequency and mode stabilization loops were started. Fig. 10 shows the time-averaged frequency errors after stabilization at a submount temperature of $20^{\circ} \mathrm{C}$, both with and without modulation. There is clearly a negative offset of the frequencies when the laser is being modulated (average offset $=-0.85 \mathrm{GHz}$ ), because the phase current stabilizes at a slightly lower value with the modulation switched on (Fig. 11). This is probably due to the frequency chirp, which alters the time-averaged signal detected by the Fabry-Perot wavelength locker. With modulation, the SMSR is generally slightly reduced, but remains above $35 \mathrm{~dB}$ for all channels (see Fig. 12). Since a constant current swing was used for all channels, the average power varied by about $5 \mathrm{~dB}$ across the different channels. A typical eye diagram is shown in Fig. 13.

For the $193.9 \mathrm{THz}$ channel, a temperature ramp was again applied. The temperature was changed from 20 to $25^{\circ} \mathrm{C}$ in 0.5

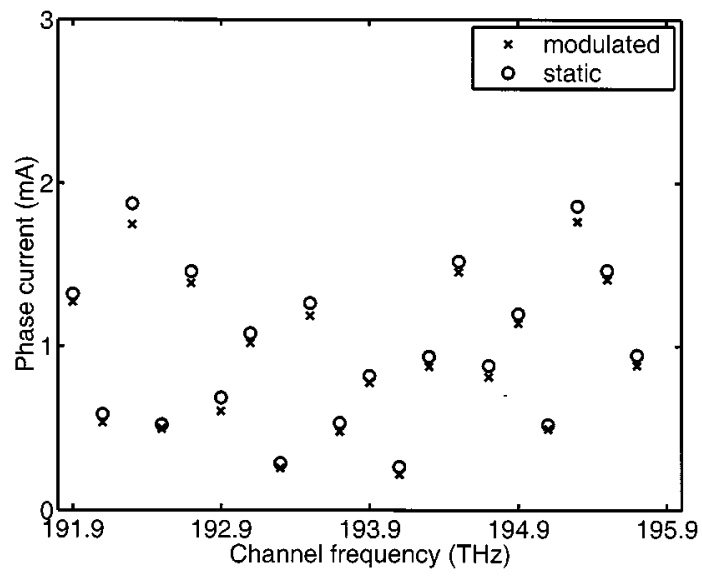

Fig. 11. Phase currents after stabilization of 20 ITU channels at $20^{\circ} \mathrm{C}$, with direct modulation at $1.244 \mathrm{~Gb} / \mathrm{s}(x)$ and in static operation (o).

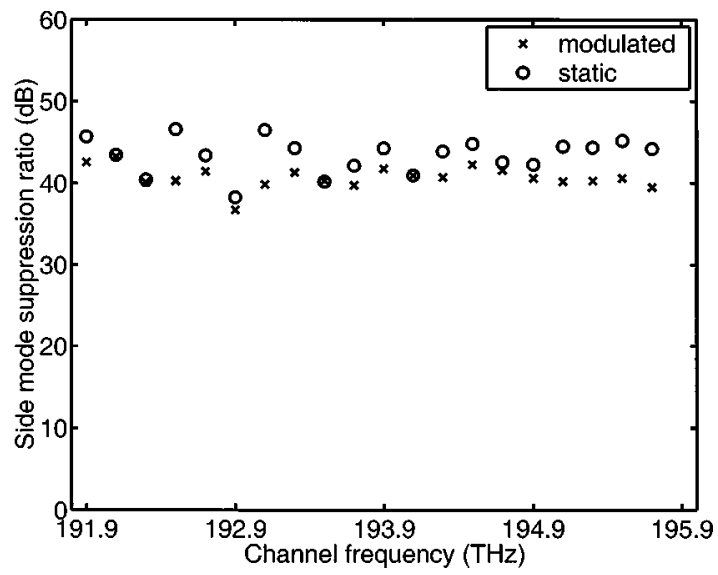

Fig. 12. Side mode suppression ratio after stabilization of 20 ITU channels at $20^{\circ} \mathrm{C}$, with direct modulation at $1.244 \mathrm{~Gb} / \mathrm{s}(x)$ and in static operation (o).

${ }^{\circ} \mathrm{C}$ steps. As previously explained, tuning currents increase with temperature and therefore the active section bias current had to be increased manually during the temperature ramp in order to maintain a good SMSR (the threshold current increases due to the additional losses). The current was increased from $39.5 \mathrm{~mA}$ at $20^{\circ} \mathrm{C}$ to $41.9 \mathrm{~mA}$ at $25^{\circ} \mathrm{C}$. The evolution of the frequency error and the temperature with time are shown in Fig. 14. After each temperature step, the frequency stabilizes again to within $\pm 0.2 \mathrm{GHz}$ of the initial frequency of $193.895 \mathrm{THz}$ (cf., Fig. 10).

\section{Generating LooK-Up TABles using the Voltage MONITORING TECHNIQUE}

\section{A. Requirements for Characterization Procedures}

Before describing the characterization procedure used to generate a look-up table for an SSG-DBR laser in detail, it is useful to briefly discuss what is in general required. The main criterion is of course frequency accuracy. As mentioned in the introduction, typically an accuracy of $\pm 10 \%$ of the channel spacing is demanded. Put the other way around, this means that the higher the accuracy, the denser the channel spacing can become. With the procedure outlined in [21] for GCSR lasers, accuracies of $\pm 3 \mathrm{GHz}$ were obtained, which is sufficient for a $50-\mathrm{GHz}$ 


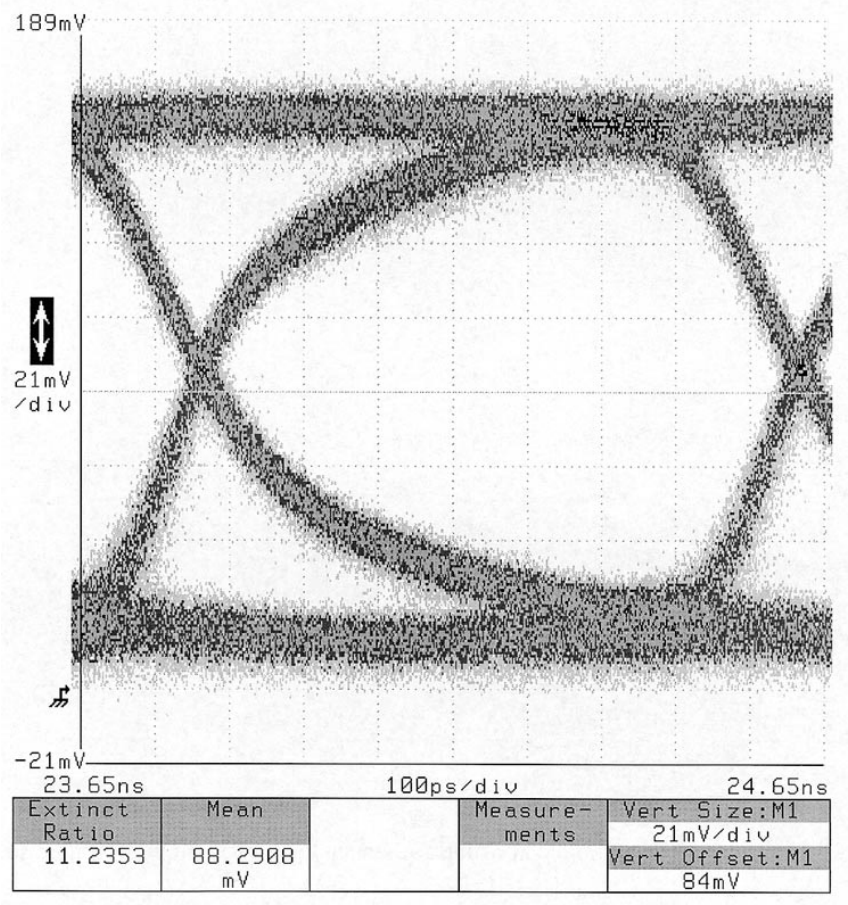

Fig. 13. Eye diagram of SSG-DBR laser stabilized at $192.7 \mathrm{THz}$, directly modulated at a bit rate of $1.244 \mathrm{~Gb} / \mathrm{s}$ with a $2^{23}-1$ PRBS signal.

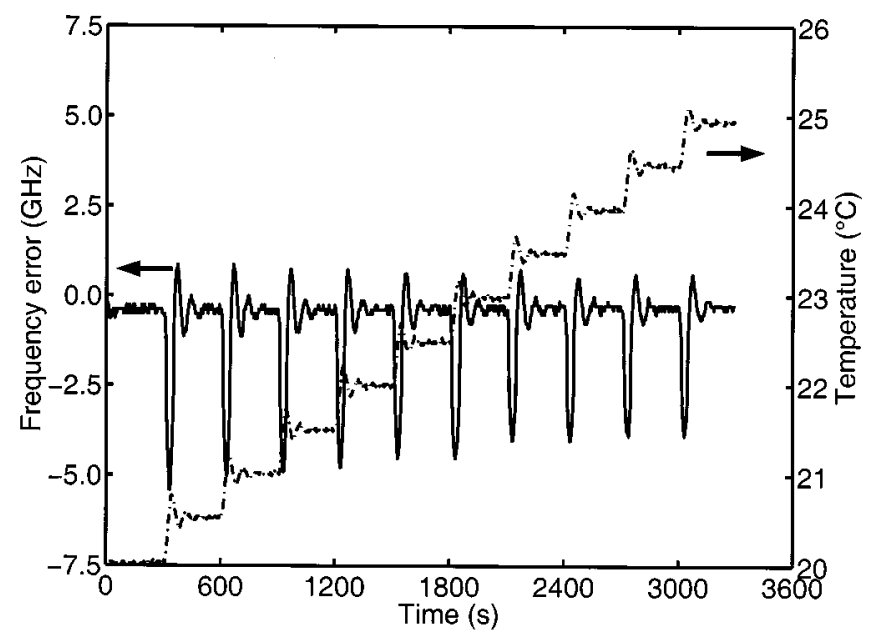

Fig. 14. Frequency and mode control applied to a directly modulated SSG-DBR laser tuned to $193.9 \mathrm{THz}$. Evolution of frequency error and submount temperature with time.

channel spacing, but not for $25 \mathrm{GHz}$. At the same time, a good side mode suppression ratio must be guaranteed. Normally, the SMSR should be at least $30 \mathrm{~dB}$.

The second main criterion is characterization speed, since this will have an important impact on the cost of the tunable transmitter module, i.e., the laser with its associated control electronics. Until recently, it typically took two to three days to generate a look-up table for an SSG-DBR laser, using a full-automatic trial-and-error procedure (table of about 400 points in 10 $\mathrm{GHz}$ increments) [22]. Concerning cost, there are also a few secondary criteria, e.g., the amount of time expensive equipment like a wavelength meter is needed, or the amount of operator intervention that is required.

\section{B. Description of the Procedure}

Our procedure basically consists of following steps. First, all the minimum voltage points in the $\left(I_{\text {front }}, I_{\text {rear }}\right)$-plane are located, for a number of different phase currents between 0 and $I_{\text {phase, } 2 \pi}$. Only in these optimum operation points, the frequency will be measured. Afterwards, the points are grouped per cavity mode and the look-up table is calculated through straightforward interpolation between the measured points. A priori knowledge of a few parameters is assumed. First, one should have an idea of the maximum front and rear DBR currents that are required to get full wavelength coverage $\left(I_{\text {front, } \max }\right.$ and $\left.I_{\text {rear, } \max }\right)$. Second, also an estimate of the phase current needed to get a $2 \pi$-phase shift is required $\left(I_{\text {phase, } 2 \pi}\right)$.

Reasonable estimates for these currents can be obtained automatically as follows. Full wavelength coverage requires that the front (rear) reflector is at least tuned over a range equal to its reflectivity peak spacing (Fig. 2). The current $I_{\text {front, max }}$ ( $\left.I_{\text {rear, max }}\right)$ needed to do so is obtained by setting the front and rear reflector currents to 0 and then gradually increasing the front (rear) reflector current, while monitoring the emission wavelength. The wavelength will then increase (decrease) stepwise with jumps of about $5 \mathrm{~nm}$ as subsequent pairs of reflector peaks become aligned (cf., Fig. 3). At a certain point, the wavelength jumps down (up) to the other end of the tuning range, after which it starts increasing (decreasing) again. The current $I_{\text {front, } \max }\left(I_{\text {rear, max }}\right)$ is reached when the wavelength again crosses its initial value (i.e., at zero tuning currents). Since only a low-resolution wavelength measurement is needed here, a simple wavelength discriminating filter can be used instead of a wavelength meter to speed up the measurement [21]. In order to have some error margin, one can increase the value of $I_{\text {front, } \max }\left(I_{\text {rear, max }}\right)$ obtained in this way by, e.g., $10 \%$.

The $2 \pi$-phase shift current can be determined by setting the phase current to zero, and initializing the reflector currents to a point of minimum active section voltage. This point can for example be obtained by setting the front and rear reflector currents to half their maximum values, and have the voltage feedback loop stabilize the reflector currents on the closest voltage minimum. Subsequently, the feedback loop is switched off and the phase current is gradually increased, while monitoring the voltage modulation components $V_{f}$ and $V_{r}$ (Fig. 6). The cavity mode will then move away from the aligned reflector peaks, and the signals $\left|V_{f}\right|$ and $\left|V_{r}\right|$ will increase. At a certain point, an adjacent cavity mode will become the preferential mode, and a mode hop will occur, after which $\left|V_{f}\right|$ and $\left|V_{r}\right|$ will decrease again. The $2 \pi$-phase shift current is reached when $\left|V_{f}\right|$ and $\left|V_{r}\right|$ reach zero again. As for the reflector currents, some error margin can be built-in by taking a value for $I_{\text {phase, } 2 \pi}$ that is somewhat larger than the value found.

In more detail, the look-up table generation algorithm then runs as follows. First, the outer edges of the tuning range are scanned to locate the super mode jumps, i.e., wavelength jumps of more than $5 \mathrm{~nm}$. The rear reflector current is set to $I_{\text {rear, }}$ max and the front reflector current is stepwise increased from 0.1 $\mathrm{mA}$ to $I_{\text {front, } \max }$. Afterwards, $I_{\text {front }}$ is kept constant and $I_{\text {rear }}$ is stepwise reduced to $0.1 \mathrm{~mA}$. In every point, the wavelength is measured at zero phase current. At present, a wavelength meter 
is used, but since the only purpose is to locate the super mode jumps, again a coarse wavelength measurement with a wavelength discriminating filter would be sufficient. In the points between two subsequent super mode jumps, the phase current is scanned from $I_{\text {phase, } 2 \pi}$ to 0 (for our lasers from 1.90 to 0.01 $\mathrm{mA}$ in $0.27 \mathrm{~mA}$ steps) and the voltage modulation components $V_{f}$ and $V_{r}$ are measured (Fig. 6). From the set of points obtained in this way, the combination of front DBR, rear DBR and phase current is picked that is situated closest to a voltage minimum in the $\left(I_{\text {front }}, I_{\text {rear }}\right)$-plane for that particular phase current. This combination is found by requiring a minimal value for the trial function

$$
T\left(I_{\text {front }}, I_{\text {rear }}, I_{\text {phase }}\right)=\sqrt{I_{\text {front }} \cdot V_{f}^{2}+I_{\text {rear }} \cdot V_{r}^{2}} .
$$

Indeed, we find for $V_{f}$ :

$$
\begin{aligned}
V_{f} & =\frac{\partial V_{\text {active }}}{\partial I_{\text {front }}} \Delta I_{\text {front }} \\
& =\frac{\partial V_{\text {active }}}{\partial \nu_{\text {front }}} \frac{\partial \nu_{\text {front }}}{\partial N_{\text {front }}} \frac{\partial N_{\text {front }}}{\partial I_{\text {front }}} \Delta I_{\text {front }} .
\end{aligned}
$$

Here $\nu_{\text {front }}$ is the frequency of the front reflector peak, $N_{\text {front }}$ is the carrier density in the front reflector section, and $\Delta I_{\text {front }}$ is the (constant) modulation component of the front reflector current. Near a voltage minimum, the variation of the active section voltage as a function of the misalignment between the cavity mode and the reflector peaks $\left(\Delta \nu_{f}\right.$ and $\Delta \nu_{r}$, respectively) can be approximated by a parabola.

$$
V_{\text {active }}=V_{0}+a\left\lfloor\left(\Delta \nu_{f}\right)^{2}+\left(\Delta \nu_{r}\right)^{2}\right\rfloor .
$$

As a result, $\partial V_{\text {active }} / \partial \nu_{\text {front }}$ is proportional to the frequency misalignment between the cavity mode and the reflector peak $\Delta \nu_{f}$.

$$
\frac{\partial V_{\text {active }}}{\partial \nu_{\text {front }}} \propto \Delta \nu_{f}
$$

Since $\partial \nu_{\text {front }} / \partial N_{\text {front }}$ can be assumed constant, and to a good approximation $I_{\text {front }} \propto\left(N_{\text {front }}\right)^{2}$, we finally obtain

$$
V_{f}=C_{f} \cdot \Delta \nu_{f} \frac{\Delta I_{\text {front }}}{\sqrt{I_{\text {front }}}} .
$$

Here $C_{f}$ is a constant. An analogous relation can be written down for $V_{r}$. If $C_{f} \approx C_{r} \approx C$ and $\Delta I_{\text {front }}=\Delta I_{\text {rear }}=\Delta I$, (1) becomes

$$
T\left(I_{\text {front }}, I_{\text {rear }}, I_{\text {phase }}\right) \approx C \Delta I \sqrt{\left(\Delta \nu_{f}\right)^{2}+\left(\Delta \nu_{r}\right)^{2}} .
$$

After the first step described above, a number of near-optimum operation points are obtained on the outer edge of the tuning range. In a second step, an iterative procedure is started for each of these near-optimum points. The tuning currents are initialized to the selected values and the voltage minimizing feedback loop is started. Following procedure is then repeated until either $I_{\text {front }}$ or $I_{\text {rear }}$ reaches the lower limit of $0.1 \mathrm{~mA}$ :

- Wait until $I_{\text {front }}$ and $I_{\text {rear }}$ have stabilized.

- Measure the frequency and store it together with the values of the different currents.

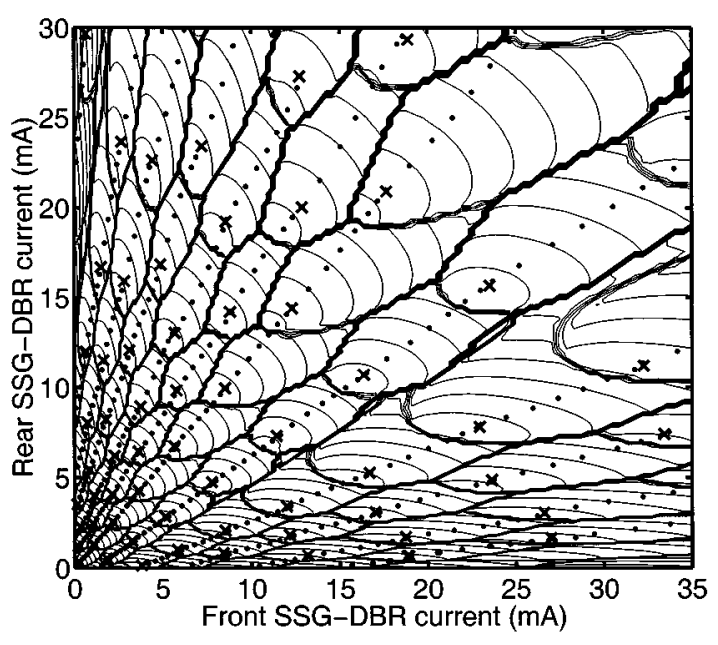

Fig. 15. Scatter plot of optimum operation points in the $\left(I_{\text {front }}, I_{\text {rear }}\right)$-plane for various phase currents $(\cdot)$ and zero phase current $(x)$, superimposed on a frequency contour map measured at zero phase current. The active section current was kept constant at $60 \mathrm{~mA}$.

- Reduce the phase current (e.g., by $0.27 \mathrm{~mA}$ ). If $I_{\text {phase }}$ would become negative, reinitialize it to $I_{\text {phase, } 2 \pi}(\approx 1.9$ $\mathrm{mA})$.

In this way, a table of optimum operation points is built up. Fig. 15 shows a scatter plot of these points in the ( $\left.I_{\text {front }}, I_{\text {rear }}\right)$-plane, superimposed on a frequency contour map measured at zero phase current (crosses indicate the operation points with $I_{\text {phase }}=0 \mathrm{~mA}$ ). The obtained points nicely line up to form "tracks" between two super mode jumps. In the 3-D space of tuning currents, they line up to tracks describing the continuous tuning of individual cavity modes. The ensemble of these cavity modes covers the entire tuning range of the device.

The final step is obviously calculating the look-up table from the measured points. This is done as follows. The operation points that were found are grouped per individual cavity mode and stored in a table together with the measured frequencies. For a particular frequency, first the cavity modes that can be tuned to this frequency are searched. The search criterion is of course that the demanded frequency should lie between the minimal and maximal frequency measured for a cavity mode. If multiple solutions are found, that cavity mode that can be tuned to the desired frequency with minimal total tuning current is selected, since a minimal sum of tuning currents will yield lowest absorption losses. Because frequency varies sublinearly as a function of the tuning currents, a cubic interpolation scheme is applied to the selected mode's measured operation points in order to calculate the tuning currents for the desired frequency.

Actually, the procedure can be taken one step further. As it is described above, the algorithm assumes a fixed active section current. This means that the look-up table that is generated will show large output power variations across the different operating points, due to the carrier-induced loss in the passive sections. For the SSG-DBR lasers, typically between 5-7 dB power variation is measured. However, since the algorithm uses feedback to find optimum operation points, one can easily add a power control loop, which runs in parallel with the voltage-minimizing loop and adjusts the active section current. In practice, 


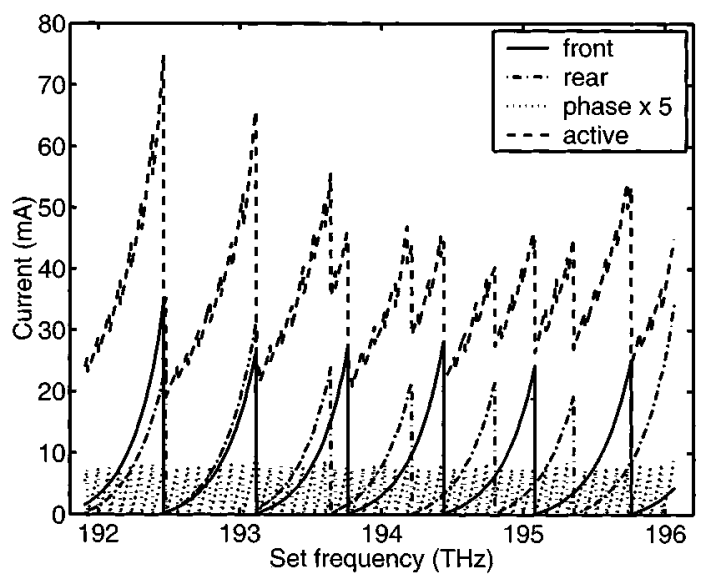

Fig. 16. Plot of an automatically generated look-up table. Currents were calculated for frequencies from 191.9 to $196.1 \mathrm{THz}$ in $1 \mathrm{GHz}$ steps.

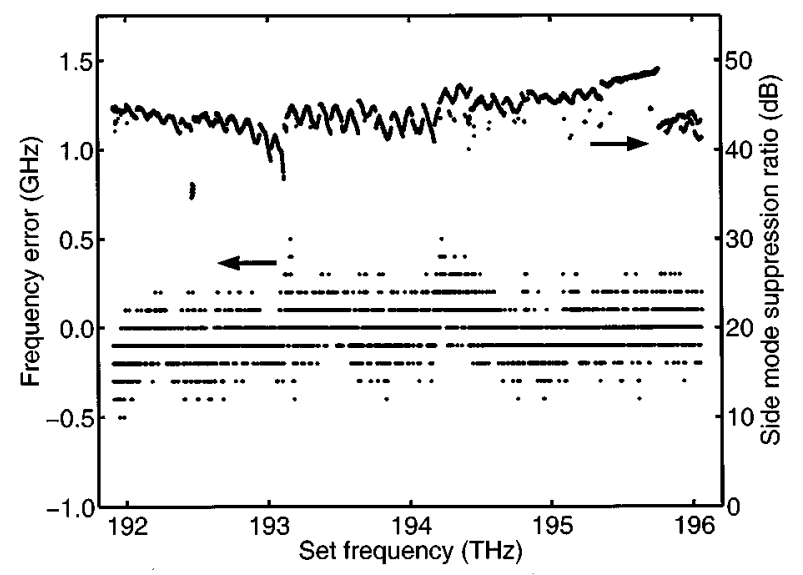

Fig. 17. Measured frequency errors and side mode suppression ratios for the look-up table plotted in Fig. 16.

we use the signal from photodiode $P_{1}$ to equalize the output power (cf., Fig. 6).

\section{Experimental Results}

Fig. 16 shows an example of a look-up table that was generated using the procedure outlined above, with power equalization included. The output power was stabilized at approximately $-3 \mathrm{dBm}$. Currents were calculated for frequencies from 191.9 to $196.1 \mathrm{THz}$ in $1 \mathrm{GHz}$ steps. There is an obvious correlation between the active section current and the three tuning currents. The active section current varies from 20 to $75 \mathrm{~mA}$. Threshold currents range from 7 to $19 \mathrm{~mA}$. When the look-up table is verified, very good frequency accuracy and side mode suppression ratio are obtained for all points. Frequency errors are less than $\pm 0.5 \mathrm{GHz}$ and the SMSR is always above $35 \mathrm{~dB}$, mostly even above $40 \mathrm{~dB}$ (Fig. 17). The root-mean-squared (rms) frequency error is typically less than $0.15 \mathrm{GHz}$. With these errors, applications in dense wavelength-division-multiplexing (DWDM) systems with channel spacings of $25 \mathrm{GHz}$ or even $10 \mathrm{GHz}$ become feasible.

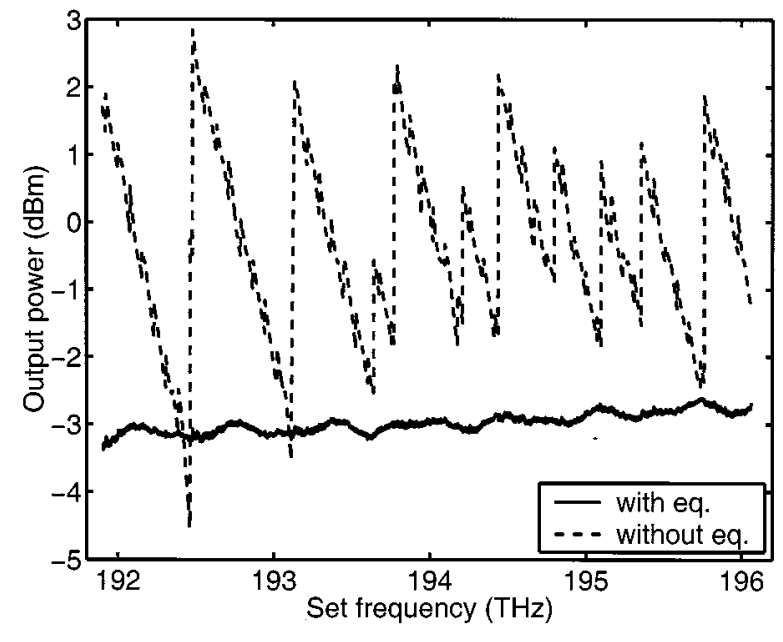

Fig. 18. Measured output powers for the look-up table plotted in Fig. 16, i.e., with power equalization (solid line), as well as for a look-up table that was generated without power equalization (dashed line). In the latter case, the active section current was fixed at $60 \mathrm{~mA}$.

The measured frequency accuracy is mostly limited by the wavelength meter used (HP 86120B multi-wavelength meter [19]). It is already clear from Fig. 17 that the resolution of the wavelength meter is only $0.1 \mathrm{GHz}$. The datasheet of the instrument specifies an absolute accuracy of $\pm 3 \mathrm{ppm}( \pm 0.6 \mathrm{GHz})$ and a differential accuracy of $\pm 2 \mathrm{ppm}( \pm 0.4 \mathrm{GHz})$. Nonetheless, one needs a very accurate temperature control, which completely eliminates variations of environmental temperature. A temperature variation of only $0.02{ }^{\circ} \mathrm{C}$ already gives a frequency drift of about $0.25 \mathrm{GHz}$. Finally, it is essential to use the current sources that will eventually drive the laser already at the characterization stage, in order to eliminate errors due to inaccuracies of the current sources as much as possible. Our present current sources have a resolution of $3 \mu \mathrm{A}$ for the phase current, and $8 \mu \mathrm{A}$ for the reflector currents. Multiplied by the tuning efficiencies at low currents this leads to frequency resolutions on the order of $0.2 \mathrm{GHz}$. When Fig. 17 is compared to Fig. 16, it is clear that the largest frequency errors are obtained when the tuning currents are lowest.

As Fig. 18 illustrates, the power variation has been reduced to less than $0.8 \mathrm{~dB}$; a reduction by almost a factor of ten compared to the situation where no power control is applied. The remaining power variation is probably due to the frequency dependence of the photodiode $P_{1}$ and the two power splitters inside the wavelength locker.

The characterization time needed to generate a full look-up table is currently about $150 \mathrm{~min}$ with power control and 100 min without. However, as already mentioned, the present feedback loop is still relatively slow, since discrete instruments are used, which are connected via GPIB and controlled by a program running on a PC. It is expected that characterization time will be drastically reduced if these instruments are replaced by electronic circuitry integrated on a single board and controlled by a microprocessor. In that case, the main limitation will be the time needed for frequency measurements. In general, the procedure generates a list of 500-600 operation points for which the frequency has to be measured. Since a frequency measurement 
typically takes 1 second, the lower limit for the characterization time is $10 \mathrm{~min}$. We estimate that with properly designed electronics, a total measurement time of less than $30 \mathrm{~min}$ is certainly feasible.

\section{CONCLUSION}

In this paper, we presented a general discussion on the control of widely tunable SSG-DBR lasers. A feedback control scheme was introduced that ensures frequency stability and accuracy (better than $\pm 0.5 \mathrm{GHz}$ ), as well as high side mode suppression ratio $(>35 \mathrm{~dB})$. The active section voltage is monitored to maintain mode stability and a highly stable Fabry-Perot etalon is used as a reference to lock the laser frequency to a specific ITU channel. It was shown that stability could even be maintained when directly modulating the laser at $1.244 \mathrm{~Gb} / \mathrm{s}$.

Furthermore, a characterization scheme was demonstrated that uses the voltage monitoring to generate a look-up table of operation points very efficiently and accurately. For all operation points, the obtained frequency accuracy was better than $\pm 0.5 \mathrm{GHz}$ and the side mode suppression ratio was above $35 \mathrm{~dB}$. With these accuracies, applications in DWDM systems with channel spacings of $25 \mathrm{GHz}$ or even less certainly become feasible. By including power equalization at the characterization stage, power variations across the tuning rage were reduced from almost $8 \mathrm{~dB}$ to only $0.8 \mathrm{~dB}$. The total characterization time was drastically reduced, from 2-3 days to 100-150 minutes. It is expected that by integrating the control circuitry on a single board, the characterization time can even be further reduced to 20-30 minutes. In practice, also some form of screening will be necessary, so as to filter out defective devices before the characterization procedure is started.

In principle, the methods described in this paper can also be applied to other three- or four-section DBR-type lasers (with minor adjustments). However, care has to be taken that the lasers are properly designed, such that for example the reflection peaks are sufficiently narrow, the carrier-induced losses are not excessively high and hysteresis is avoided as much as possible.

\section{ACKNOWLEDGMENT}

The authors would like to thank all the partners in the EU Project ACTS-AC329 ACTUAL for the many productive discussions, especially Dr. H. Ishii and Dr. Y. Yoshikuni of NTT Optoelectronics Labs, who also supplied the SSG-DBR lasers.

\section{REFERENCES}

[1] K. Kobayashi and I. Mito, "Single frequency and tunable laser diodes," J. Lightwave Technol., vol. 6, pp. 1623-1633, Nov. 1988.

[2] M.-C. Amann and J. Buus, Tunable Laser Diodes. Norwood, MA: Artech House, 1998.

[3] F. Delorme, S. Slempkes, G. Alibert, B. Rose, and J. Brandon, "Buttjoined DBR laser with $15 \mathrm{~nm}$ tunability grown in three MOVPE steps," Electron. Lett., vol. 31, no. 15, pp. 1244-1245, 1995.

[4] M. Öberg, S. Nilsson, K. Streubel, L. Bäckbom, and T. Klinga, "74 nm wavelength tuning range of an InGaAsP/InP vertical grating assisted codirectional coupler laser with rear sampled grating reflector," IEEE Photon. Technol. Lett., vol. 5, pp. 735-738, July 1993.

[5] P.-J. Rigole, S. Nilsson, L. Bäckbom, B. Stålnacke, E. Berglind, J.-P Weber, and B. Stoltz, "Quasicontinuous tuning range from 1560 to 1520 nm in a GCSR laser, with high power and low tuning currents," Electron. Lett., vol. 32, no. 25, pp. 2352-2354, 1996.
[6] P.-J. Rigole, S. Nilsson, L. Bückbom, B. Stålnacke, T. Klinga, E. Berglind, B. Stoltz, D. J. Blumenthal, and M. Shell, "Wavelength coverage over $67 \mathrm{~nm}$ with a GCSR laser. Tuning characteristics and switching speed," in Proc. XV IEEE Int. Semiconductor Laser Conf., Haifa, Israel, 1996, Paper W1.1.

[7] J. Willems, G. Morthier, and R. Baets, "Novel widely tunable integrated optical filter with high spectral selectivity," in Proc. ECOC'92, Berlin, Germany, 1992, Paper WeB9.2.

[8] V. Jayaraman, Z. M. Chuang, and L. A. Coldren, "Theory, design and performance of extended tuning range semiconductor lasers with sampled gratings," IEEE J. Quantum Electron., vol. 29, pp. 1824-1834, June 1993.

[9] F. Delorme, "Widely tunable $1.55-\mu \mathrm{m}$ lasers for wavelength-divisionmultiplexed optical fiber communications," IEEE J. Quantum Electron., vol. 34, pp. 1706-1716, Sept. 1998.

[10] H. Ishii, H. Tanobe, F. Kano, Y. Tohmori, Y. Kondo, and Y. Yoshikuni, "Quasicontinuous wavelength tuning in super- structure-grating (SSG) DBR lasers," IEEE J. Quantum Electron., vol. 32, pp. 433-440, Mar. 1996.

[11] _ "Broad-range wavelength coverage $(62.4 \mathrm{~nm})$ with superstructuregrating DBR laser," Electron. Lett., vol. 32, no. 5, pp. 454-455, 1996.

[12] B. Villeneuve, M. Cyr, and H.B. Kim, "High-stability wavelength-controlled DFB laser sources for dense WDM applications," Proc. OFC'98, 1998.

[13] S. L. Woodward, V. Mizrahi, T. L. Koch, U. Koren, and P. J. Lemaire, "Wavelength stabilization of a DBR laser using an in-fiber Bragg filter," IEEE Photon. Technol. Lett., vol. 5, pp. 628-630, June 1993.

[14] Y. Park, S.-T. Lee, and C.-J. Chae, "A novel wavelength stabilization scheme using a fiber Bragg grating for WDM transmission," IEEE Photon. Technol. Lett., vol. 10, pp. 1446-1448, Oct. 1998.

[15] H. Ishii, F. Kano, Y. Yoshikuni, and H. Yasaka, "Mode stabilization method for superstructure-grating DBR lasers," J. Lightwave Technol., vol. 13, pp. 433-442, Mar. 1998.

[16] G. Sarlet, G. Morthier, and R. Baets, "Wavelength and mode stabilization of widely tunable SG-DBR and SSG-DBR lasers," IEEE Photon. Technol. Lett., vol. 11, pp. 1351-1353, Nov. 1999.

[17] S. L. Woodward, T. L. Koch, and U. Koren, "A control loop which ensures high side-mode-suppression ratio in a tunable DBR laser," IEEE Photon. Technol. Lett., vol. 4, pp. 417-419, May 1992.

[18] 95131E-Tek Dynamics Inc., Fabry-Perot Wavelength Locker (FPWL), San Jose, CA.

[19] 5301 Stevens Creek Blvd.95052Agilent Technologies, Test \& Measurement, HP 86 120B multi-wavelength meter, Santa Clara, CA, USA.

[20] G. Sarlet, H. Ishii, Y. Yoshikuni, G. Morthier, and R. Baets, "Direct modulation properties of widely tunable lasers,", submitted for publication.

[21] T. Farrell, J. Dunne, and R. O'Dowd, "Complete wavelength control of GCSR lasers over EDFA band," in Proc. LEOS'99, San Francisco, CA, 1999, Paper TuY1.

[22] H. Ishii and Y. Yoshikuni, private communication.

Gert Sarlet (S'99) was born in Antwerp, Belgium, on July 10, 1973. He received the electronic engineering degree from the University of Gent, Belgium, in 1996. He is currently working toward the Ph.D. degree in electronic engineering at the Department of Information Technology (INTEC), University of Gent-IMEC.

During his engineering studies, he spent one year as an exchange student at the Swiss Federal Institute of Technology (ETH), Zürich, Switzerland. He is currently a Research Assistant with the Fund for Scientific Research-Flanders (Belgium). His main research interests are the design, the control, and the dynamic behavior of widely tunable lasers.

Geert Morthier (M'93) received the degree in electrical engineering and the Ph.D. degree from the University of Gent, Belgium, in 1987 and 1991, respectively.

Since 1991, he is a member of the permanent staff of IMEC. His main interests are in the modeling and characterization of optoelectronic components. He has authored or coauthored over 70 papers in the field. He is also one of the two authors of the "Handbook of Distributed Feedback Laser Diodes" (Artech House, 1997) and coeditor of the book "How to model and measure photonic components: experience from a European project" (Springer-Verlag, 1998). From 1998 to end of 1999, he has been the Project Manager of the EU ACTS project ACTUAL dealing with the control and applications of widely tunable laser diodes. 
Roel Baets (M'88-SM'96) received the degree in electrical engineering from the University of Gent, Belgium, in 1980. He received the M.Sc. degree in electrical engineering from Stanford University, Stanford, CA, in 1981 and the Ph.D. degree from the University of Gent in 1984.

Since 1981, he is with the Department of Information Technology (INTEC) of the University of Gent. Since 1989, he has been a Professor in the engineering faculty of the University of Gent. From 1990 to 1994, he was also a part-time Professor at the Technical University of Delft, The Netherlands. He has worked in the field of III-V devices for optoelectronic systems. With about 200 publications and conference papers he has made contributions to the design and fabrication of semiconductor laser diodes, passive guided wave devices, PICs and microoptic components. He leads the Optoelectronic Components and Systems group at the University of Gent-IMEC, working on photonic devices for optical communication and optical interconnect.

Dr. Baets is a member of the Optical Society of America (OSA), SPIE, and the Flemish Engineers Association. He has been member of the program committees of OFC, ECOC, IEEE Semiconductor Laser Conference, ESSDERC, CLEO-Europe and the European Conference on Integrated Optics. Currently he is Chairman of the IEEE-LEOS Benelux chapter. 